LBNL-62259

January 2007

\title{
Space-Charge Effects in the Super B-Factory LER *
}

\author{
M. Venturini \\ Lawrence Berkeley National Laboratory, \\ Berkeley, CA, 94720 (USA)
}

\begin{abstract}
Space-charge effects in the low-energy ring of the proposed Super-B Factory are studied using a weak-strong model of dynamics as implemented in the code Marylie/Impact (MLI). The impact of space charge appears noticeable but our results suggest the existence of workable regions of the tune space where the design emittance is minimally affected. However, additional studies are recommended to fully substantiate this conclusion.
\end{abstract}

*Work supported by Department of Energy contract DE-AC02-05CH11231 
A large bunch population and small beam sizes will result in appreciable space-charge tuneshifts in the Super B-Factory rings, and in particular in the LER, as space charge effects scale inversely with the beam energy. For the LER at the design equilibrium and bunch population $\left(N=6.16 \times 10^{10}\right)$ linear theory $(i=x, y)$

$$
\Delta \nu_{i}=-\frac{1}{4 \pi} \frac{2 r_{e}}{\beta^{2} \gamma^{3}} \int_{0}^{C} \frac{\lambda \beta_{i}}{\sigma_{i}\left(\sigma_{x}+\sigma_{y}\right)} d s
$$

yields the following horizontal and vertical space-charge tuneshifts: $\Delta \nu_{x}=-0.004, \Delta \nu_{y}=$ -0.179 . The above equation, where $\beta$ and $\gamma$ are the relativistic factors, $\beta_{x}, \beta_{y}$ are the lattice functions, $\sigma_{x}, \sigma_{y}$ the horizontal and vertical rms beam sizes, $\lambda=N / \sqrt{2 \pi} \sigma_{z}$ the longitudinal peak density ( $\sigma_{z}$ is the rms longitudinal bunch length), applies to particle undergoing infinitesimally small betatron and synchrotron oscillations about the center of a gaussian bunch. Plots of the transverse beam sizes for LER at equilibrium as determined using the design emittances $\varepsilon_{x}=0.71 \mathrm{~nm}, \varepsilon_{y}=2.5 \mathrm{pm}$, and $\varepsilon_{z}=5 \mu \mathrm{m}$ are reported in Fig. 1 .

While space charge should be of little consequence on injection efficiency, as its effects become noticeable only after a few damping times, it could cause particle beam losses at later times if the working point in tune-space is sufficiently close to an unstable lattice resonance. Proximity to stable resonances would be less damaging but could also be detrimental and lead to unacceptable emittance degradation. Far from resonances space charge may still compromise the achievement of the target vertical equilibrium emittance when its impact is considered in combination with radiation and linear coupling in an non-ideal lattice. This latter effect, however, should be small [1] and was neglected here, as we limited our attention to an error-free lattice in the absence of any radiation effect.

Our study was conducted using a weak-strong model for space charge, as implemented in the numerical tools recently developed to study similar effects in the ILC damping rings $[2,3]$. In the weak-strong model the space charge force is calculated as if produced by a $6 \mathrm{D}$ gaussian bunch matched to the ideal linear lattice and with rms emittances equal to those expected at equilibrium for a realistic lattice with some residual linear coupling. A collection of macroparticles, initially distributed according to a bunch density at equilibrium,
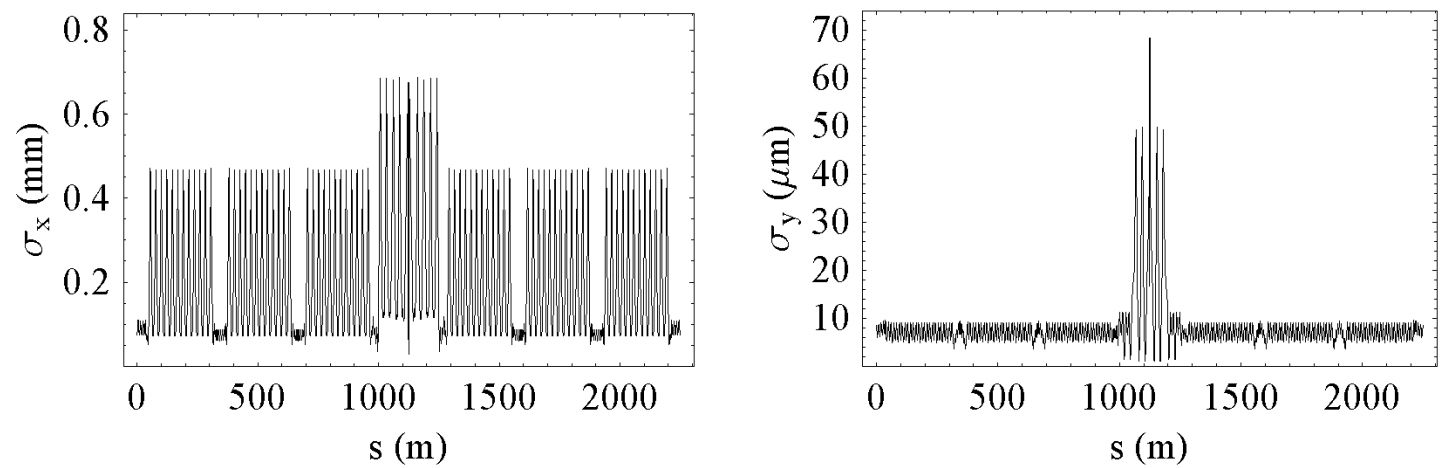

Figure 1: Rms transverse beam sizes along the LER lattice at equilibrium. The horizontal size (left picture) includes the effect of a finite energy spread in the dispersive regions. 

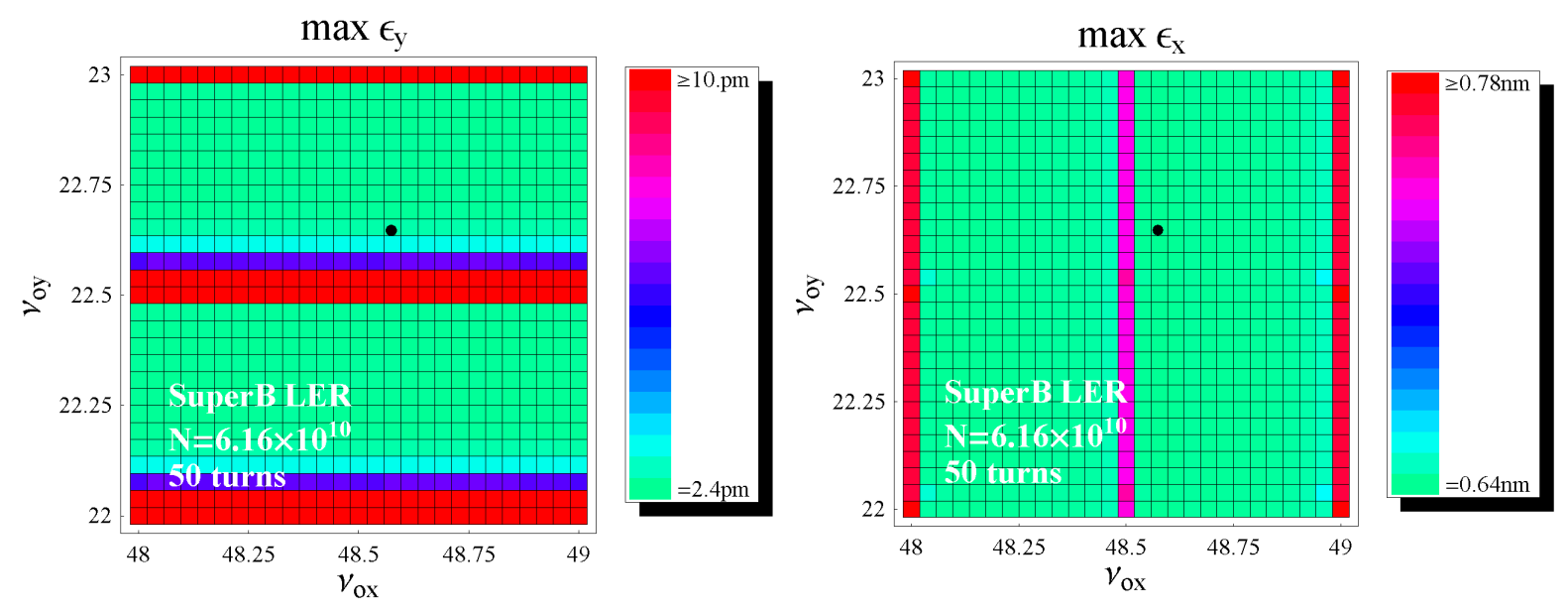

Figure 2: Tune scan of horizontal and vertical max. rms emittance growth over 50 machine turns with space charge. Here and in the following figures we used a linear scale for the max. emittances color-coding function. The design working point $\nu_{0 x}=48.575, \nu_{0 y}=48.647$ is shown as a black dot.

is then tracked element by element with the inclusion of the lattice nonlinearities and spacecharge force applied in the kick approximation. Because of its non-self consistent nature, this model will likely overestimate the effect of any detected emittance growth and should be used mainly as a tool to search the tune space for regions of minimal emittance growth. An accurate characterization of emittance growth would require more detailed and considerably more computationally intensive models of beam dynamics.

In our study we used an augmented version of Marylie/Impact (MLI) code [5]. The code was validated during the ILC damping ring studies by calculations carried out independently using $\mathrm{SAD}[4,2]$. For more details on the implementation of the weak-strong space-charge model in MLI we refer to [2].

We assessed the space-charge effects in the LER lattice by producing tune space scans and looking for the rms emittance changes in the transverse plane. The results of our investigations are reported as color-density plots showing the maximum value of the rms emittance experienced by the macroparticle beam within the indicated duration of tracking, see Figs. 2, 3, and 4. We tracked a bunch population of 200 macroparticles. Because of the weak-strong nature of the model, evaluation of the space charge kick is independent of the number of macroparticles used and a modest number should be sufficient to provide an acceptable sampling of the phase space available to the beam. The detuning of the lattice was done by inserting pure phase rotations at the end of the one-turn lattice with proper matching so as not to perturb the value of the lattice functions. This amounts to a linear kick causing a small discontinuity in both the particle transverse position and momentum.

A short term tracking (50 machine turns) tune scan of the $[0,1] \times[0,1]$ region around the design working point detected strong half-integer resonances (Fig. 2). These resonances are already present in the bare lattice and the effect of space charge is to noticeably enlarge 

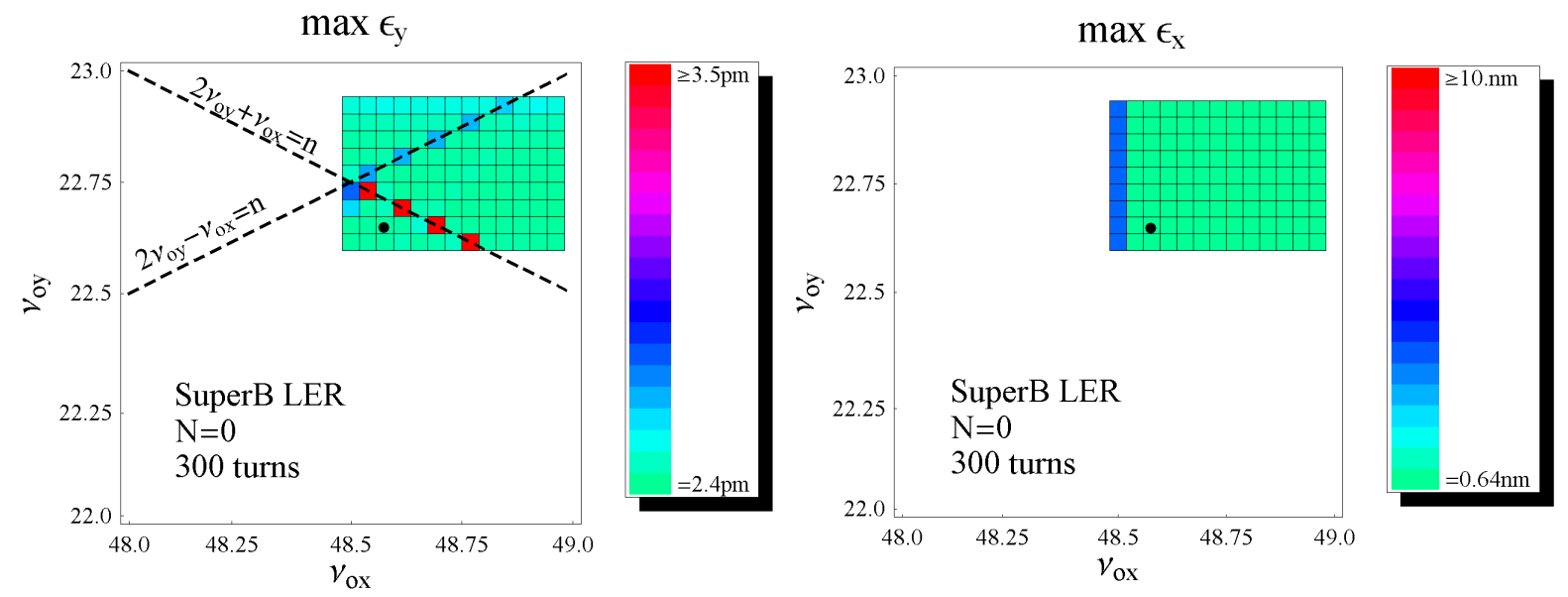

Figure 3: Tune scan of horizontal and vertical max. rms emittance growth over 300 machine turns without space charge.

their width. This is seen in the vertical plane, left picture in Fig. 2, where the apparent width of the resonance lines is about $\Delta \nu_{y} \simeq 0.1$. Notice, however, that such a value is somewhat smaller than the tuneshift predicted by linear theory. This should be interpreted as a consequence of the highly nonlinear nature of the space charge force, which causes the tuneshift experienced by particles to decrease quickly down from the value predicted by linear theory with increasing amplitude of the betatron (or synchrotron) oscillations. In the horizontal plane (right picture in Fig. 2) the space charge tuneshift is too small to have any detectable effect on the scale of resolution used for these tune-scans. Incidentally, notice that the minimum emittance reported in the pictures, $\varepsilon_{y}=2.4 \mathrm{pm}$ and $\varepsilon_{x}=0.65 \mathrm{~nm}$ (corresponding to dark green in the color coding), representing the rms emittances of the actual realizations of the initial macroparticle distribution, are slightly different from the nominal equilibrium values $\varepsilon_{y}=2.5 \mathrm{pm}$ and $\varepsilon_{x}=0.71 \mathrm{~nm}$ because of statistical fluctuations due to the use of a limited number of macroparticles.

For practical reasons, to study space-charge effects on a longer time scale (up to 600 machine/turns) we restricted our investigation to a smaller area of tune-space. The case with (Fig. 3) is contrasted with the case without (Fig. 4) space charge. In the absence of space charge the vertical emittance tune scan (left picture in Fig 3) shows evidence of two third-order resonances at $2 \nu_{0 y}+\nu_{0 x}=n$ and $2 \nu_{0 y}-\nu_{0 x}=n$, with the first being considerably stronger and resulting into about $100 \%$ emittance growth over 300 machine turns. The other resonance resulted into a smaller $10 \%$ growth over the same tracking time. Outside these narrow resonances the vertical rms emittance appear to remain substantially invariant.

Inclusion of phase charge causes some additional degradation of the rms vertical emittance not apparent in short term-tracking (left picture in Fig. 4). Not unexpectedly, the largest growth occurs along the half-integer $\nu_{0 x}=48.5$ line. This resonance is already present in a bare lattice but with visible consequences only on the horizontal motion. Its impact on the vertical motion is fostered by the $x / y$ coupling introduced by space-charge. The emittance 

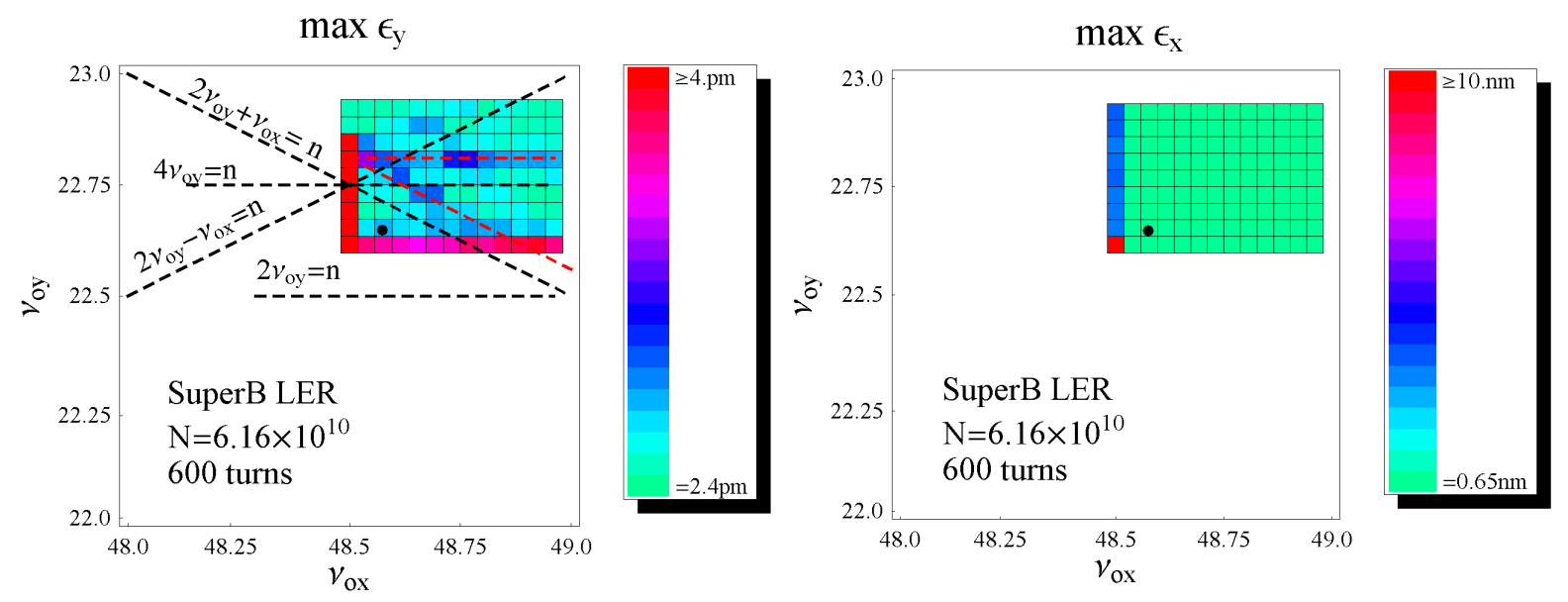

Figure 4: Tune scan of horizontal and vertical max. rms emittance growth over 600 machine turns with space charge.

growth detected along this line was very large and for some choices of the vertical tune was found to lead to particle losses. Outside this resonance line and the upper part of the region affected by the $\nu_{0 y}=22.5$ resonance we observe some smaller but clearly noticeable emittance growth up to about $30 \%$ over 600 turns (the bluish region of the plot). The emittance growth appears to be concentrated mostly along two lines - we highlighted these strips of growth in the left picture of Fig. 2 by two red dashed lines. These lines are not traceable to some obvious resonances and identification of the exact growth mechanism will require some more work. At this time our tentative interpretation is that these regions of growth are related to the resonance lines $2 \nu_{0 y}+\nu_{0 x}=n$ and $4 \nu_{0 y}=n$, and appear shifted upwardly for reasons to be further investigated. Notice that the magnitude of this shift is well within the value of space charge detuning - an argument in favor of this interpretation. The resonance $2 \nu_{0 y}+\nu_{0 x}=n$ is a lattice resonance already present in the bare lattice whereas the fourth order $4 \nu_{0 y}=n$ could be ascribed to a space-charge induced resonance caused by the space-charge force nonlinearity and the breathing of the the beam envelopes. The latter is one of the lowest-order nonlinear resonances that can be driven by space charge.

In conclusion, this first preliminary study indicates that space charge effects are noticeable in the low energy ring. One clear consequences is the enlargement of strong half-integer structural lattice resonances present in the bare lattice, causing a fast emittance growth and possibly particle losses. This alone poses a significant limitation to the choice of the working point because of the sizeable space-charge vertical tuneshift. On a longer time scale we encountered some areas of moderate but clearly detectable emittance growth. Encouragingly, however, our calculations also show the existence regions in the tune space that appear little affected by emittance growth. Further studies will be needed to insure that motion stability would persist on a longer timescale (up to a few damping times) and in the presence of lattice errors. 


\section{References}

[1] M. Venturini, K. Oide, and A. Wolski, Space-Charge and Equilibrium Emittances in Damping Rings, LBNL Report no. LBNL-59509, (2006) Berkeley, (also in EPAC-06 Proceedings).

[2] M. Venturini and K. Oide, Direct Space-Charge Effects on the ILC Damping Rings: Task Force Report, LBNL Report no. LBNL-59511 (2006).

[3] J. Gao, S. Guiducci, and A. Wolski Editors, Recommendations for the ILC Damping Rings Baseline Configuration, LBNL Report LBNL-59449, Berkeley (2006).

[4] SAD Home page: http://acc-physics.kek.jp/SAD

[5] A. Dragt, et al., MARYLIE Manual, University of Maryland, College Park, MD (2004). 
This document was prepared as an account of work sponsored by the United States Government. While this document is believed to contain correct information, neither the United States Government nor any agency thereof, nor The Regents of the University of California, nor any of their employees, makes any warranty, express or implied, or assumes any legal responsibility for the accuracy, completeness, or usefulness of any information, apparatus, product, or process disclosed, or represents that its use would not infringe privately owned rights. Reference herein to any specific commercial product, process, or service by its trade name, trademark, manufacturer, or otherwise, does not necessarily constitute or imply its endorsement, recommendation, or favoring by the United States Government or any agency thereof, or The Regents of the University of California. The views and opinions of authors expressed herein do not necessarily state or reflect those of the United States Government or any agency thereof, or The Regents of the University of California.

Ernest Orlando Lawrence Berkeley National Laboratory is an equal opportunity employer. 